Documentation et bibliothèques

\title{
La formation des bibliothécaires, des archivistes et des documentalistes au Sénégal
}

\section{Bernard Dione}

Volume 61, numéro 2-3, avril-septembre 2015

La formation en sciences de l'information

URI : https://id.erudit.org/iderudit/1032818ar

DOI : https://doi.org/10.7202/1032818ar

Aller au sommaire du numéro

Éditeur(s)

Association pour l'avancement des sciences et des techniques de la documentation (ASTED)

ISSN

0315-2340 (imprimé)

2291-8949 (numérique)

Découvrir la revue

Citer cet article

Dione, B. (2015). La formation des bibliothécaires, des archivistes et des documentalistes au Sénégal. Documentation et bibliothèques, 61(2-3), 117-120. https://doi.org/10.7202/1032818ar

Tous droits réservés (C) Association pour l'avancement des sciences et des techniques de la documentation (ASTED), 2015
Ce document est protégé par la loi sur le droit d'auteur. L'utilisation des services d'Érudit (y compris la reproduction) est assujettie à sa politique d'utilisation que vous pouvez consulter en ligne.

https://apropos.erudit.org/fr/usagers/politique-dutilisation/ 


\title{
La formation des bibliothécaires, des archivistes et des documentalistes au Sénégal
}

\author{
BernaRd Dione, Ph. D. \\ Enseignant \\ Directeur des études \\ École de bibliothécaires, archivistes et documentalistes (EBAD) \\ Université Cheikh Anta Diop de Dakar \\ bernard.dione@ucad.edu.sn
}

\section{Introduction}

L

'École de bibliothécaires, archivistes et documentalistes $(\mathrm{EBAD})^{1}$ est la seule école chargée de former les bibliothécaires, les archivistes et les documentalistes au Sénégal. Elle a également été, pendant plusieurs années, la principale école de formation en sciences de l'information en Afrique subsaharienne.

\section{Le Centre régional de formation de bibliothécaires}

L'EBAD est un institut d'université créé par le décret $n^{\circ}$ 67-1235 du 17 novembre 1967 en remplacement $\mathrm{du}$ Centre régional de formation de bibliothécaires des pays d'Afrique d'expression française (CRFB), qui a fonctionné à Dakar de novembre 1963 à juin 1967. La création du CRFB a été le fruit d'un long processus. L'idée de créer une école de bibliothéconomie en Afrique francophone subsaharienne remonte au Séminaire sur le développement des bibliothèques publiques en Afrique, organisé à Ibadan, au Nigeria, en 1953. Lors de ce séminaire, la précarité de la situation des bibliothèques, aussi bien dans les pays de l'ancienne Afrique occidentale française (AOF) et de l'Afrique-Équatoriale française (AÉF) qu'à Madagascar et au Congo, avait été mise en exergue. L'une des solutions préconisées contre cette précarité était de créer une école de bibliothéconomie capable de former le personnel scientifique et technique des bibliothèques des pays d'Afrique francophone subsaharienne.

En outre, lors de la conférence des chefs d'États africains sur le développement de l'éducation en Afrique, tenue en 1961 à Addis Abeba (Éthiopie), les participants, dont principalement l'UNESCO, ont insisté sur la création d'un grand nombre de bibliothèques qu'il faudrait pourvoir en bibliothécaires qualifiés et ont exprimé la nécessité de créer une école de bibliothéconomie.

C'est dans cette perspective que l'UNESCO a décidé, en 1961, de créer à Dakar le CRFB. Laccord d'installation du Centre à Dakar a été signé le 28 mars 1962 par

1. Voir <www.ebad.ucad.sn/index.html $>$.
l'UNESCO et la République du Sénégal (Séguin 1964). Cet accord stipulait en son article 2, paragraphe 1, que les cours du CRFB devraient bénéficier, outre aux ressortissants de la République du Sénégal, à ceux du Cameroun, de la République centrafricaine, de la République du Tchad, de la République populaire du Congo, du Dahomey (actuel Bénin), du Gabon, de la Guinée, de la Côte d'Ivoire, du Mali, de la Mauritanie, du Niger, du Togo, de la Haute-Volta (actuelle Burkina Faso), du Zairre (actuelle République démocratique du Congo), de Madagascar, du Rwanda et du Burundi. Le démarrage du CRFB au cours de l'année universitaire 1963-1964 a marqué les débuts de l'enseignement de la bibliothéconomie en Afrique francophone.

\section{L'École de bibliothécaires, archivistes et documentalistes}

Le concours de l'UNESCO a pris fin en juin 1967. Le gouvernement sénégalais, ayant estimé qu'il fallait faire face à la pénurie de personnel qualifié qui entravait l'organisation et le développement des bibliothèques, des services d'archives et des centres de documentation, a décidé du maintien et du développement d'une école de formation professionnelle pour la préparation aux fonctions de bibliothécaire, d'archiviste et de documentaliste. La délégation sénégalaise à la Commission mixte franco-sénégalaise en matière d'enseignement supérieur a proposé à la France de s'associer à la transformation du CRFB en institut d'université, prenant le nom d'École de bibliothécaires, archivistes et documentalistes (EBAD 1972).

Selon le décret $\mathrm{n}^{\circ}$ 2007-1206 du 8 octobre 2007 portant son organisation et son fonctionnement, l'EBAD a pour missions essentielles :

- la formation initiale et continue des bibliothécaires, archivistes et documentalistes et de tout autre professionnel relevant des sciences de l'information documentaire, en présentiel et à distance;

- la recherche en sciences de l'information documentaire pour le développement d'une expertise africaine francophone; 
- l'étude et l'implantation de systèmes et services d'information au profit d'institutions publiques et/ ou privées [...]. (Sénégal. Ministère de l'Éducation)

L'EBAD hérite du CRFB son caractère régional. De 1979 à 2009, elle aura formé 2551 bibliothécaires, archivistes et documentalistes en Afrique subsaharienne et en Haïti. Le Tableau 1 donne une idée juste de son apport important dans la formation des bibliothécaires africains et indique que l'origine des étudiants a même débordé le cadre de l'Afrique francophone subsaharienne. L'école a formé des professionnels de l'information venant de pays lusophones (Cap-Vert, Guinée-Bissau, Guinée équatoriale) et anglophones (Gambie, Sierra Léone).

Jusqu'aux années 1970, l'EBAD a été effectivement la seule école de formation spécialisée en sciences de l'information de niveau universitaire en Afrique francophone au sud du Sahara. Elle est d'ailleurs toujours la seule école de sciences de l'information au Sénégal.

\section{Tableau 1}

Diplômés de l'EBAD par pays, par section et par cycle de 1979 à 2009 (EBAD. Statistiques des diplômés)

\begin{tabular}{|c|c|c|c|c|c|c|c|c|}
\hline \multirow[t]{2}{*}{ Pays } & \multicolumn{2}{|c|}{ Archivistique } & \multicolumn{2}{|c|}{ Bibliothéconomie } & \multicolumn{2}{|c|}{ Documentation } & \multirow[t]{2}{*}{ Total } & \multirow[t]{2}{*}{$\%$} \\
\hline & $1^{\mathrm{er}}$ cycle & $2^{\mathrm{e}}$ cycle & $1^{\mathrm{er}}$ cycle & $2^{\mathrm{e}}$ cycle & $1^{\mathrm{er}}$ cycle & $2^{\mathrm{e}}$ cycle & & \\
\hline Bénin & 19 & 1 & 15 & 2 & 12 & 8 & 57 & $2,23 \%$ \\
\hline Burkina Faso & 31 & 5 & 29 & 0 & 45 & 12 & 122 & $4,78 \%$ \\
\hline Burundi & 3 & 1 & 8 & 1 & 3 & 0 & 16 & $0,63 \%$ \\
\hline Cameroun & 38 & 4 & 47 & 3 & 48 & 7 & 147 & $5,76 \%$ \\
\hline Cap-Vert & 7 & 5 & 0 & 0 & 7 & 5 & 24 & $0,94 \%$ \\
\hline Centrafrique & 21 & 0 & 12 & 0 & 20 & 0 & 53 & $2,08 \%$ \\
\hline Comores & 12 & 0 & 1 & 0 & 5 & 0 & 18 & $0,71 \%$ \\
\hline Congo & 5 & 0 & 9 & 0 & 20 & 0 & 34 & $1,33 \%$ \\
\hline Côte d'Ivoire & 33 & 4 & 43 & 0 & 46 & 2 & 128 & $5,02 \%$ \\
\hline Gabon & 35 & 2 & 13 & 2 & 46 & 5 & 103 & $4,04 \%$ \\
\hline Gambie & 2 & 0 & 0 & 0 & 0 & 0 & 2 & $0,08 \%$ \\
\hline Guinée-Bissau & 1 & 0 & 2 & 0 & 1 & 0 & 4 & $0,16 \%$ \\
\hline Haute-Volta & 12 & 0 & 14 & 0 & 33 & 0 & 59 & $2,31 \%$ \\
\hline Madagascar & 4 & 0 & 5 & 2 & 7 & 7 & 25 & $0,98 \%$ \\
\hline Mali & 3 & 0 & 11 & 5 & 15 & 1 & 35 & $1,37 \%$ \\
\hline Niger & 13 & 0 & 9 & 1 & 40 & 0 & 63 & $2,47 \%$ \\
\hline Rwanda & 8 & 0 & 14 & 1 & 6 & 0 & 29 & $1,14 \%$ \\
\hline Sénégal & 375 & 55 & 392 & 67 & 398 & 89 & 1376 & $53,94 \%$ \\
\hline Seychelles & 1 & 0 & 0 & 0 & 0 & 0 & 1 & $0,04 \%$ \\
\hline Tchad & 11 & 5 & 11 & 3 & 37 & 5 & 73 & $2,86 \%$ \\
\hline Togo & 48 & 6 & 32 & 6 & 50 & 7 & 149 & $5,84 \%$ \\
\hline Zaïre & 3 & 1 & 11 & 0 & 2 & 0 & 17 & $0,67 \%$ \\
\hline Mauritanie & 0 & 0 & 2 & 0 & 3 & 0 & 5 & $0,20 \%$ \\
\hline Haïti & 0 & 0 & 1 & 0 & 0 & 0 & 1 & $0,04 \%$ \\
\hline Djibouti & 0 & 0 & 1 & 0 & 4 & 0 & 5 & $0,20 \%$ \\
\hline Guinée équatoriale & 0 & 0 & 2 & 1 & 0 & 0 & 3 & $0,12 \%$ \\
\hline Sierra Leone & 0 & 0 & 1 & 0 & 1 & 0 & 2 & $0,08 \%$ \\
\hline TOTAL & 629 & 75 & 633 & 76 & 784 & 124 & 2551 & $100,00 \%$ \\
\hline
\end{tabular}




\section{Les programmes de formation}

Le programme de formation de l'EBAD a beaucoup évolué. À l'origine, l'école était chargée de former des techniciens capables de gérer les bibliothèques publiques. Elle s'enrichit, en 1971, d'une section Archives, puis d'une section Documentation en 1975 (Correa \& Sagna 1999). L'EBAD conserve encore cette spécificité française qui sépare bibliothécaires et documentalistes et dont la pertinence, même en France, " est régulièrement interrogée et continue de susciter des débats très passionnés au sein de professions qui se sentent fortement remises en cause par les nouvelles pratiques induites par l'usage des documents numériques et des recherches sur la Toile» (Wiegandt 2005).

Les études furent d'abord organisées autour d'un $1^{\text {er }}$ cycle de deux ans qui accueillait des étudiants titulaires d'un baccalauréat en enseignement secondaire. Le cursus était sanctionné par un diplôme d'aptitude aux fonctions de bibliothécaire, d'archiviste ou de documentaliste. Un $2^{\mathrm{e}}$ cycle fut créé en 1983 . Le niveau de recrutement était alors la licence ou un diplôme de $1^{\text {er }}$ cycle de l'EBAD avec trois années d'expérience professionnelle. À l'issue de deux années de formation, les diplômés devenaient conservateurs de bibliothèques, de centres de documentation et d'archives, titres hérités de la culture bibliothéconomique française (Bats 2012; Diakhaté 2014).

À partir de 2000, grâce au projet FORCIIR (FORmations continues en informations informatisées en réseaux) financé par la coopération française, l'EBAD offre son programme de $2^{\mathrm{e}}$ cycle à distance, ce qui lui permet de consolider sa volonté d'intégration sousrégionale. Le projet FORCIIR offrira également à l'EBAD l'opportunité de développer les compétences de ses étudiants dans les technologies de l'information (Sow \& Tendeng 2001; Loiret 2007; Diakhaté 2014).

En 2004, le Sénégal adopte la réforme Licence Master Doctorat (LMD). Cette réforme est consacrée par la Loi $\mathrm{n}^{\circ}$ 2011-05 du 30 mars 2011 relative à l'organisation du système LMD dans les établissements d'enseignement supérieur qui précise aux articles 3 et 4 que :

[l]e système LMD est organisé en semestres. Dans chaque semestre peuvent être acquis 30 crédits de formation, capitalisables et transférables dans et entre les universités, et en unités d'enseignement qui correspondent chacune à une ou plusieurs disciplines. Un crédit est estimé à au moins 20 heures de travail ou d'enseignement [...]. La licence [sic] est découpée en 6 semestres et est validée par 180 crédits correspondant à 03 années d'études au moins après le baccalauréat [...]. Le Master est organisé en 4 semestres et est validé par 120 crédits après la Licence. Il totalise 300 crédits, soit 5 années d'études au moins après le baccalauréat.

(Sénégal 2011)

L'EBAD s'est adaptée à cette nouvelle architecture de la formation. Ainsi, son $1^{\mathrm{er}}$ cycle est désormais sanctionné par une licence professionnelle en sciences de l'information documentaire qui remplace les diplômes de niveau $\mathrm{Bac}+2$, avec des matières encore plus exhaustives et plus à même de répondre aux exigences du marché. Au $2^{\mathrm{e}}$ cycle, le diplôme de master en sciences de l'information documentaire remplace l'ancien diplôme supérieur en sciences de l'information et de la communication (DSSIC) (Diakhaté 2014).

La licence professionnelle en sciences de l'information documentaire (LIPSID) est structurée en six semestres de 30 crédits chacun, pour un total de trois ans et 180 crédits. Un crédit correspond à 20 heures de travail réparties en heures de cours et en heures de travail personnel de l'étudiant. Le programme conserve toujours la structuration en trois spécialités (bibliothèques, archives et documentation), bien que certains cours (description bibliographique, recherche et diffusion de l'information, informatique documentaire, etc.) soient offerts en tronc commun afin de permettre aux diplômés de s'adapter aux réalités du marché de l'emploi, lequel est de plus en plus défavorable. La faiblesse du réseau des bibliothèques et l'absence d'une politique nationale de lecture publique affectent particulièrement le recrutement. Mis à part les bibliothèques universitaires, les bibliothèques publiques dignes de ce nom sont plutôt rares. La Bibliothèque nationale du Sénégal n'existe que d'un point de vue légal. Les institutions publiques recrutent très peu de bibliothécaires en ce moment.

Quant au master en sciences de l'information, il est offert avec trois spécialités : (1) Valorisation du patrimoine documentaire; (2) Ingénierie documentaire; (3) Technologie de l'information. Il est structuré en quatre semestres de 30 crédits chacun, pour un total de 120 crédits sur deux ans. À la fin de la formation, l'étudiant doit réaliser un projet professionnel ou présenter un mémoire de recherche.

Depuis l'instauration de la réforme LMD, toutes les formations de l'enseignement supérieur au Sénégal doivent être évaluées et habilitées par l'Autorité nationale d'assurance qualité de l'enseignement supérieur (ANAQ-Sup), l'agence de l'État du Sénégal chargée de l'habilitation et de l'accréditation des programmes et des institutions d'enseignement supérieur.

L'ANAQ-Sup ${ }^{2}$ a été créée par le décret $n^{\circ}$ 2012-837 du 7 août 2012. Placée sous la tutelle administrative et technique du ministère de l'Enseignement supérieur et de la Recherche du Sénégal, elle est chargée de contrôler, de garantir et d'améliorer la qualité des programmes

2. Voir <www.anaqsup.sn $>$. 
et des institutions d'enseignement supérieur. Au terme d'un processus d'évaluation dont la durée estimée est de deux à six mois, elle produit un rapport d'évaluation et donne une décision d'accréditation. Lorsqu'elle est accordée, l'accréditation est valable pour une durée de cinq ans. La décision peut cependant être assortie de conditions. En août 2013, l'agence a produit trois documents méthodologiques fortement inspirés des référentiels de l'agence d'assurance qualité suisse OAQ : un référentiel d'évaluation des programmes, un référentiel d'évaluation des institutions et un guide d'autoévaluation à l'intention des institutions d'enseignement supérieur du Sénégal (CAMES 2014, 47-51). (-)

\section{Sources consultées}

Bats, Raphaëlle. 2012. Former des bibliothécaires et documentalistes en français. Bulletin des bibliothèques de France 57 (6). <bbf. enssib.fr/consulter/bbf-2012-06-0022-005.pdf> (consulté le 19 avril 2015).

CAMES. 2014. Les pratiques d'assurance qualité au Sénégal. In Appui à l'assurance qualité de l'enseignement supérieur et de la recherche dans les pays de l'espace CAMES : état des lieux dans les pays de l'UÉMOA. <www.lecames.org/attachments/ article/2/EtatLieuxAQ_EUMOA_Ed2014.compressed.pdf> (consulté le 19 avril 2015).

Diakhaté, Djibril. 2014. Introduction pionnière du e-learning à l'université et son rôle dans l'élaboration de nouvelles compétences technologiques : le cas de l'EBAD. Knowledge Management for Development Journal 1o (2) : 19-32. <journal. $\mathrm{km} 4 \mathrm{dev}$. org/index.php/km4dj/article/viewFile/227/281> (consulté le 19 avril 2015).

École de bibliothécaires, archivistes et documentalistes (EBAD). 1972. Sénégal d'aujourd'hui Magazine. Numéro spécial : Formation des cadres moyens supérieurs, 20-25.

École de bibliothécaires, archivistes et documentalistes (EBAD). s.d. Statistiques des diplômés. <www.ebad.ucad.sn/diplomes/statistiques.html> (consulté le 19 avril 2015).

Loiret, Pierre-Jean. 2007. L'enseignement à distance et le supérieur en Afrique de l'Ouest : une université façonnée de l'extérieur ou renouvelée de l'intérieur? [Thèse de doctorat]. Université de Rouen. <tel.archives-ouvertes.fr/tel-oo192921/document> (consulté le 17 avril 2015).

Seguin, L. 1964. Le Centre régional de formation de bibliothécaires de Dakar. Bulletin de l'UNESCO à l'intention des bibliothèques 17 (3) : 109-118.

Sénégal. Ministère de l'Éducation. 2008 (12 janvier). Décret nº 20071206 du 8 octobre 2007 portant organisation et fonctionnement de l'École des bibliothécaires, archivistes et documentalistes (EBAD). Journal officiel (6385). <www.jo.gouv.sn/spip. php?article6484> (consulté le 17 avril 2015).

Sénégal. 2011 (25 juin). Loi n 2011-05 du 30 mars 2011 relative à l'organisation du système LMD dans les établissements d'enseignement supérieur. Journal officiel (6598). <www.jo.gouv.sn/ spip.php?article9074> (consulté le 4 juin 2015).

Sow, Mody \& Antoine Tendeng. 2001. Les nouvelles technologies en Afrique : l'expérience de l'EBAD en matière d'enseignement à distance. Communication présentée dans le cadre du Colloque Initiatives 2001, Beyrouth. <www.lb.refer.org/initiatives/document/acteo5.doc> (consulté le 19 avril 2015).

Wiegandt, Caroline. 2005. Bibliothécaires et documentalistes : deux métiers qui se rapprochent. Bulletin des bibliothèques de France (5). <bbf.enssib.fr/consulter/bbf-2005-05-0016-005> (consulté le 22 avril 2015). 